\title{
Retrograde intrarenal surgery for a staghorn renal calculus in a patient with solitary kidney and urinary tract deformity: a case report
}

\author{
Yuancheng $\mathrm{Xu}^{1}$, Zhengquan $\mathrm{Lu}^{1}$, Yulong $\mathrm{Lan}^{2}$, Dan $\mathrm{Wu}^{3}$, Lin Xiong ${ }^{1}$ \\ ${ }^{1}$ Department of Urology, The University of Hong Kong- Shenzhen Hospital, Shenzhen, China; ${ }^{2}$ Department of Cardiology, The Second Affiliated \\ Hospital of Shantou University Medical College, Shantou, China; ${ }^{3}$ Department of Pediatrics, The Second Affiliated Hospital of Shantou University \\ Medical College, Shantou, China \\ Correspondence to: Lin Xiong. Department of Urology, The University of Hong Kong-Shenzhen Hospital, 01 Haiyuan Road, Shenzhen 518053, \\ China. Email: xiongling1966@163.com.
}

\begin{abstract}
Staghorn renal calculi are large, branched stones in the kidney that partially or completely fill the renal pelvis and renal calyces. Percutaneous nephrolithotomy (PCNL) is the gold standard treatment for staghorn calculi. However, we report a retrograde intrarenal surgery (RIRS) performed to treat a staghorn calculus in a patient with a solitary kidney and a deformed urinary tract. The 37-year-old male patient presented with right-sided lumbar pain. The computed tomography (CT) scan found a solitary kidney on the right side with an opaque $4.5 \mathrm{~cm} \times 2.4 \mathrm{~cm}$ renal stone and grade I hydronephrosis. Additionally, a urinary tract deformity was observed, and it was secondary to the deformity of the pelvis caused by a previous pubis fracture, which significantly increased the risk and the difficulty of intrarenal surgery. A total number of 3 sessions of RIRS were conducted, and the patient was discharged 3 days after each session on average. The postoperative X-ray exam of the third session revealed that the renal stone was completely removed. The patient recovered well without any complications. This case demonstrates that RIRS is a safe and effective treatment of staghorn calculi with the presence of urinary tract deformation. This suggests RIRS may be of particular interest in minimizing the procedure-related damage of a solitary kidney.
\end{abstract}

Keywords: Staghorn calculus; retrograde intrarenal surgery; solitary kidney; deformity of the pelvis; percutaneous nephrolithotomy; case report

Submitted Mar 06, 2021. Accepted for publication Jul 21, 2021.

doi: $10.21037 /$ tau-21-192

View this article at: https://dx.doi.org/10.21037/tau-21-192

\section{Introduction}

Nephrolithiasis is a prevalent illness that affects $5 \%$ of the population in the United States (1), with $10 \%$ to $20 \%$ of these cases being staghorn calculi (2). The invasive procedures to treat this disease include percutaneous nephrolithotripsy (PCNL) and retrograde intrarenal surgery (RIRS). PCNL has been the first-line treatment of staghorn calculi (3), and RIRS is recommended by guidelines as the first-line treatment for kidney stones $\leq 2.0 \mathrm{~cm}$ (4). Although RIRS has been reported to have a lower stone-free rate compared to PCNL when managing kidney stones larger than $2.0 \mathrm{~cm}(5)$, the past few decades have seen technological advances and refinements that have substantially improved the stone-clearance rate of multisession RIRS. As a result, it has become increasingly popular in the treatment of larger stones $>2.0 \mathrm{~cm}$, including staghorn stones (6). Most notably, RIRS is less invasive and has a low rate of intraoperative and postoperative complications, mainly urinary tract infection and fever (7). Consequently, RIRS may become the sole option due to general health conditions and patient preference (8). By contrast, performing PCNL to manage staghorn calculi has been significantly associated with intraoperative bleed of the kidney on account of large calculi and multiple percutaneous 
punctures. Another severe complication is renal collecting system injury (up to $8 \%$ ), which may result in electrolyte disorder, changes of mental status, and intravascular volume overload (9). Therefore, RIRS is an excellent alternative for patients who prefer to undergo a safer procedure or who may suffer experience consequences from PCNL but still expect to achieve adequate efficacy. This is especially suited for patients with a solitary kidney. Nevertheless, it may be difficult for patients to undergo RIRS when deformity of the pelvis and urinary tract is present (10). However, the application of flexible ureteroscopy together with holmium laser technology, instead of a fiberoptic ureteroscope, is favorable to navigating through the deformed and distorted urinary tract.

Although Diri et al. reported that PCNL was still the recommended first-line treatment for staghorn calculus, other treatments such as RIRS can be considered to be a multimodal therapy in certain circumstances (11). Shi et al. reported that patients with a solitary kidney were more likely to develop acute kidney injury after PCNL compared to patients with normal bilateral kidneys (12). Kuroda et al. reported that RIRS should be considered a first-choice treatment for renal stones in solitary kidney patients based on the high stone-free rates, low risk of severe complications, and the preservation of renal function (13). Herein, this case report describes a rare and complex case of RIRS, which was successfully performed on a patient diagnosed with a solitary kidney and staghorn calculus combined with a distorted urinary tract.

We present the following article in accordance with the CARE reporting checklist. (available at http://dx.doi. org/10.21037/tau-21-192)

\section{Case presentation}

A 37-year-old male patient was referred to the outpatient clinic of urology with right-sided lumbar pain for 1 year. His abdominal computed tomography (Figure 1) displayed 3 findings: the left kidney and left ureter were absent (Figure $1 A, C$ ), a kidney calculus $4.5 \mathrm{~cm} \times 2.4 \mathrm{~cm}$ in size was extending from the right renal pelvis to the lower pole (Figure 1D), and the right ureter was deformed and distorted due to the pelvic deformity (Figure 1B). The lab test showed that the renal function was normal (creatinine $77.6 \mu \mathrm{mol} / \mathrm{L}$, blood urea nitrogen $5.4 \mathrm{mmol} / \mathrm{L})$. The patient had lost his left leg and left kidney, and had suffered a pelvis fracture in a car accident 10 years prior. PCNL is recommended for kidney stones larger than $2.0 \mathrm{~cm}$ and was therefore preferable. Furthermore, the deformity of the ureter, which attributed to the deformity of the pelvis, significantly increased the difficulty of performing RIRS. However, PCNL might have resulted in severe complications, especially the intraoperative bleeding of the renal parenchyma, renal collecting system injury, and renal dysfunction (9). These potential consequences made us and the patient cautious about adopting PCNL, with particular concern given to protecting the remaining right kidney. By contrast, RIRS was a safer and less invasive option. The surgical team thoroughly communicated with the patient and explained the respective advantages and disadvantages of PCNL and RIRS. Additionally, we emphasized the difficulty of conducting RIRS due to the deformed urinary tract and the possibility of reoccurrence of kidney stones due to the patient's sedentary lifestyle and deformed urinary pathway. The patient showed a strong preference for RIRS. With careful consideration and long-term planning, we also recommended the RIRS procedure. Finally, the patient was scheduled for RIRS.

The operation was performed under general anesthesia and in the lithotomy position. Under the guidance of a fluoroscope (Ziehm 8000, Ziehm Imaging GmbH, Nuremberg, Germany), a 0.038-inch hydrophilic material coated flexible tip guidewire (BARD, Limerick, Ireland) was inserted. It carefully passed the urethra, bladder, and the deformed ureter to reach the urine-collecting part of the kidney. After some careful and repeated attempts, our experienced urologist managed to navigate the flexible guidewire through the distorted and deformed parts of the ureter (Figure 1B). A ureteral access sheath (UAS, 9.5 Fr, Cook Medical, Bloomington, IN, USA) was inserted via the guidewire with fluoroscopic guidance. The laser probe was placed through a flexible renoscope (Storz Flex-X2, Tuttlingen, Germany), and the lithotripsy was performed with a laser (SphinX $30 \mathrm{~W}$, holmium-YAG laser, LISA Laser Products-OHG, Germany), with a pulse energy of $0.6-1.2 \mathrm{~J}$, at a frequency of $10-20 \mathrm{~Hz}$, a pulse peak power of $15 \mathrm{~kW}$, and $200 \mu \mathrm{m}$ of laser fiber. When image clarity was disrupted, a pumped irrigation set (irrigation $\mathrm{Y}$ set) intermittently provided pressurized fluid. A $26-\mathrm{cm}$ 4.7 Fr double-j (DJ) catheter was inserted. A single mucosal tear (approximately $1 \mathrm{~mm}$ ) of the lower renal calyx, likely caused by the laser, was observed and noted as a minor intraoperative complication. The kidney, ureter, and bladder (KUB) $\mathrm{x}$-ray showed that the staghorn calculus was gradually removed by the 3 sessions of RIRS (Figure 2). The $1^{\text {st }}$ operation lasted for 101 minutes. A postoperative 

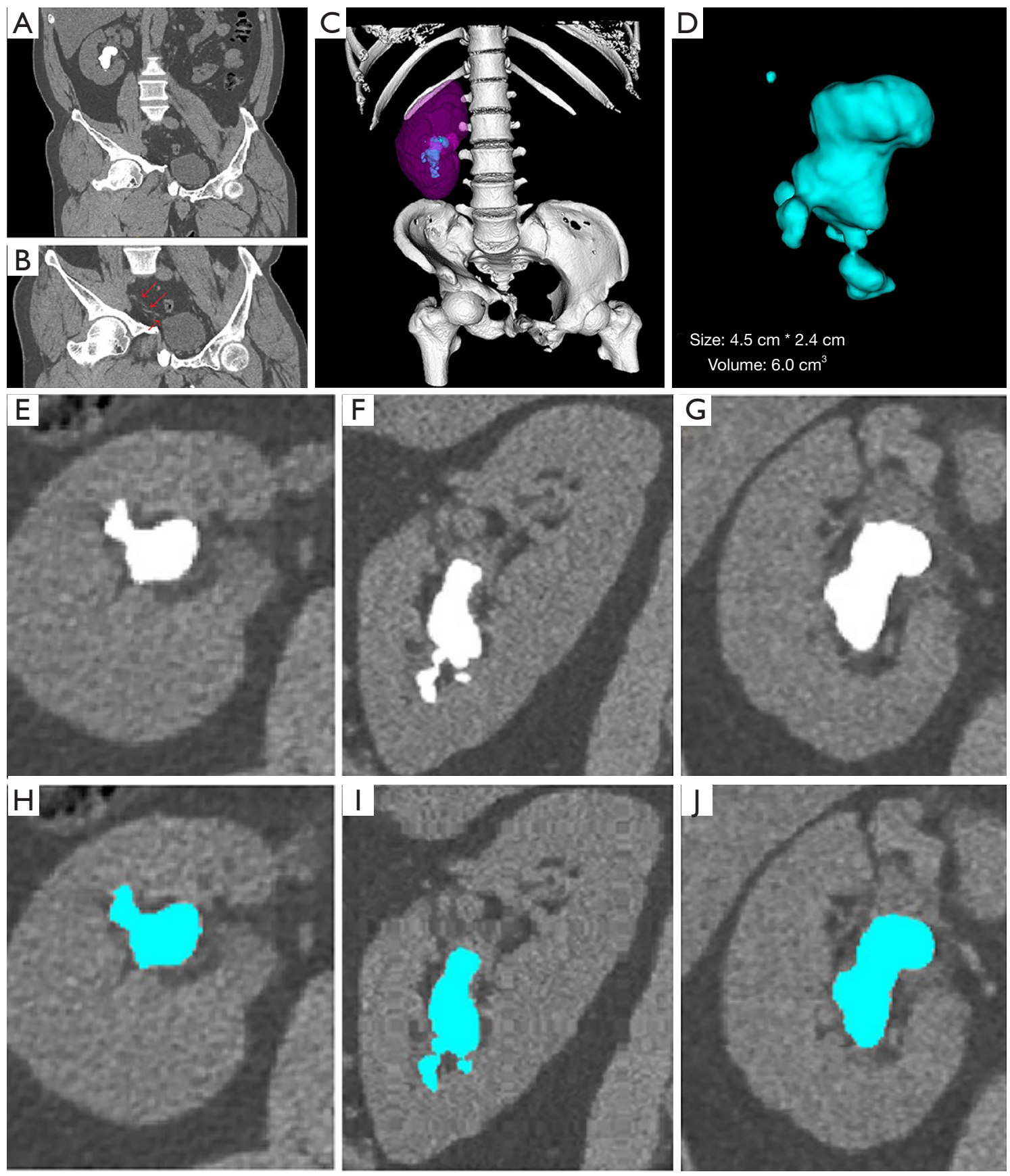

Figure 1 Computerized tomography (CT) before the first session of retrograde intrarenal surgery (RIRS). (A and B) CT before the first session of RIRS in the coronal axis showing the staghorn calculi in the solitary kidney on the right side, evident pelvic deformity (A), and a deformed and distorted urinary tract (red arrows in B). (C) Three-dimensional segmentation of the solitary kidney (purple), staghorn calculus (light blue), and bone (white) of the lower rib cage, spinal column, and pelvis. The staghorn calculus almost fully occupies the lower half of the renal collecting system of the solitary kidney. The pelvis appears deformed. (D) The amplification of the 3D segmentation of the staghorn stone. (E-J) Display of the staghorn stone in the segmented in axial (E and H), coronal (F and I), and sagittal (G and J) axis. 

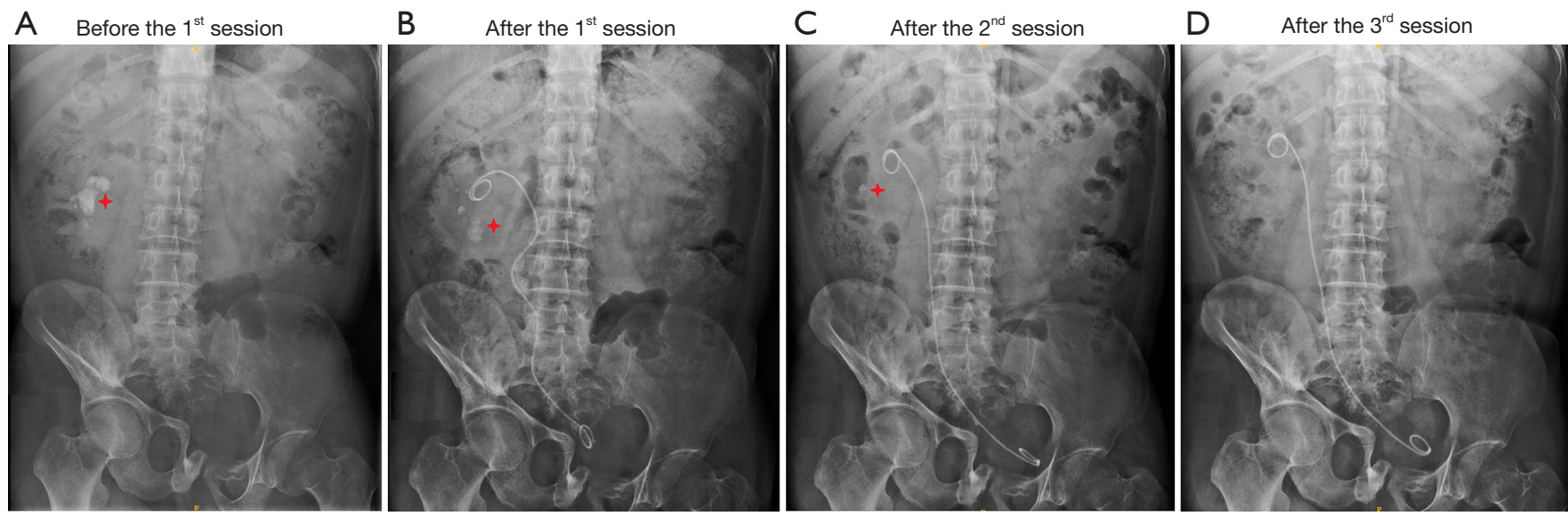

Figure 2 Serial kidney, ureter, and bladder (KUB) x-rays demonstrate the stepwise effects of 3 sessions of retrograde intrarenal surgery (RIRS) on removing the Staghorn calculus. (A) KUB before the first session of RIRS showing the staghorn calculi (red star) in the solitary kidney on the right side. (B) KUB x-rays after the first session of RIRS showing the residual stone (red star) in the solitary kidney on the right side (including the double-j stent). (C) KUB x-rays after the second session of RIRS showing the residual stone (red star) in the solitary kidney on the right side (including the double-j stent) decreased significantly compared with the first session of RIRS. (D) KUB $x$-rays after the third session of RIRS showing that there are not renal stone in the solitary kidney on the right side (including the double-j stent).

kidney, ureter, and bladder (KUB) $\mathrm{x}$-ray showed that the DJ catheter was in place (Figure 2B). All 3 sessions of RIRS were performed with an interval of approximately 20 days. The other 2 operation sessions lasted for 130 minutes and 114 minutes, respectively. The staghorn calculus was removed clearly after the $3^{\text {rd }}$ operation (Figure $2 D$ ). The physical examination and the vital signs of the patient were stable both during and after the surgeries. The level of hemoglobin remained stable throughout all 3 sessions, which was $166 \mathrm{~g} / \mathrm{L}$ before the first session, $162 \mathrm{~g} / \mathrm{L}$ after the first session, $166 \mathrm{~g} / \mathrm{L}$ after the second session, and $154 \mathrm{~g} / \mathrm{L}$ after the third session. The renal function was also consistently stable, with the creatinine level at $77.6 \mu \mathrm{mol} / \mathrm{L}$ before the first session, $79.8 \mu \mathrm{mol} / \mathrm{L}$ after the first session, $79.9 \mu \mathrm{mol} / \mathrm{L}$ after the second session, and $79.2 \mu \mathrm{mol} / \mathrm{L}$ after the third session. We segmented the staghorn calculus in 2 serial abdominal CT images (Figure 3). The CT scan in coronal axis showed that the residual renal stone were much smaller than before the second session (Figure $3 A$ ). The double-j stent and the catheter were in position (Figure $3 B$ ). The volume of the stone was $6.0 \mathrm{~cm}^{3}$ in the CT scan before the first session (Figure 1D), and $0.5 \mathrm{~cm}^{3}$ in the CT scan after the second session (Figure 3C). The amplification of the 3D segmentation of the residual stone and double- $j$ stent were displayed in the segmented axial, coronal, and sagittal axis (Figure 3D-3I). The postoperative X-ray of kidney, ureter, and bladder (KUB) of the last session of RIRS found no residual stone (Figure 2D).

All procedures performed in studies involving human participants were in accordance with the ethical standards of the institutional and/or national research committee(s) and with the Helsinki Declaration (as revised in 2013). Written informed consent was obtained from the patient.

\section{Discussion}

The treatment of solitary renal stones is still challenging. The choice of procedure should be balanced with the stonefree rates and complications, along with the need for further ancillary procedures (14). PCNL is recommended as the first-line treatment for staghorn calculi (3). Although this procedure has a higher clearance rate of stones than does RIRS (15), its higher level of invasiveness may result in serious complications, especially damage to the kidneys (16). Percutaneous access to PCNL is the main cause of damage to the renal parenchyma, the renal collecting system, and the adjacent structures, which may be critical or even lethal in patients with a solitary kidney (17). Furthermore, the PCNL procedure may induce bleeding requiring blood transfusion, septicemia, colon injury, fever, or urinary infection(18). Bleeding requiring transfusion is one of the most common complications, with an incidence rate of up to $45 \%$. 

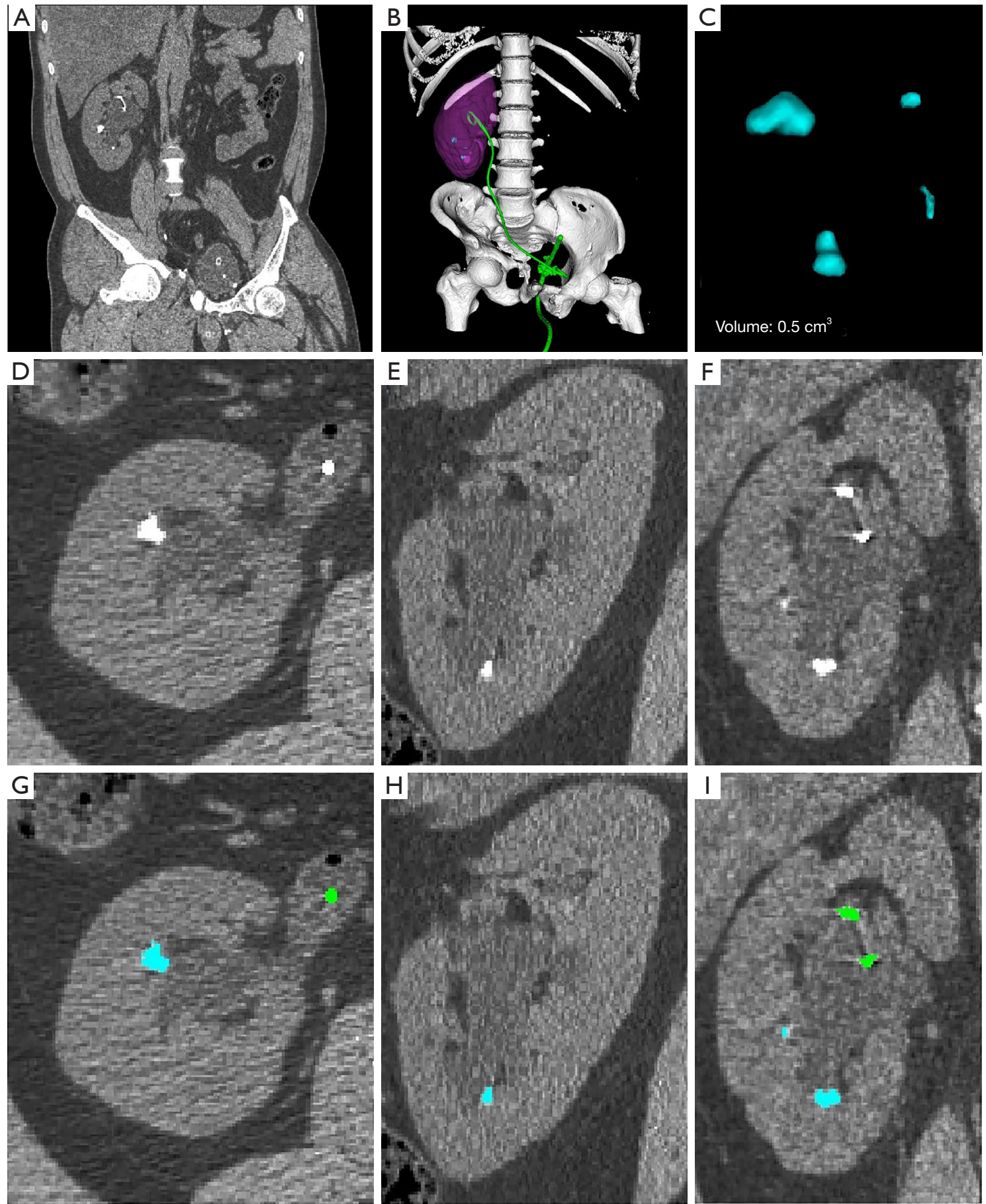

Figure 3 Computerized tomography (CT) after the second session of retrograde intrarenal surgery (RIRS). (A) CT after the second session of RIRS in coronal axis showing the residual renal stone in the solitary kidney on the right side (including the double-j stent). (B) Threedimensional segmentation of the solitary kidney (purple), residual renal stone (light blue), and the bone (white) of the lower rib cage, spinal column, and pelvis. Most of the calculus was removed after the second session of the RIRS. The double-j stent and the catheter (in green) are in position. (C). The amplification of the 3D segmentation of the remaining stone. (D-I) Display of residual stone and double-j stent in the segmented axial (D and $\mathrm{G})$, coronal (E and $\mathrm{H})$, and sagittal (F and I) axis. 
RIRS is recommended for the treatment of kidney stones smaller than $2.0 \mathrm{~cm}$. However, with the technical improvements in flexible ureteroscopy, multisessional RIRS with holmium laser lithotripsy has become an effective and safe choice for larger renal stones (19), with a stoneclearing rate comparable with that of PCNL (20). Breda et al. reported an overall stone-free rate of $92.2 \%$ after 2 sessions of RIRS for renal calculi larger than $2.0 \mathrm{~cm}$ (21). Riley et al. reported an overall stone-free rate of $90.9 \%$ after an average of 1.82 sessions of RIRS for a mean stone size of $3.0 \mathrm{~cm}$ (20). The predominant complications of RIRS reported are minor for the management of large renal stones, and mainly include postsurgical fever and pain (8). In recent years, PCNL combined with RIRS has been reported to treat staghorn calculi in cases with a solitary kidney (22) and has demonstrated reasonable safety and efficacy. Scotland et al. reported using RIRS alone to treat large renal stones, including staghorn calculi, which achieved a 3 -session stone-free rate of $94 \%$ (6). Nevertheless, RIRS has several disadvantages, including the high retreatment rate and higher cost due to the replacement and repair of flexible ureteroscopy (8). Notably, the prerequisite for removing kidney stones using RIRS is the successful navigation through the urethra and urinary tract to reach the renal pelvis.

In the case presented here, the patient had a complex medical situation manifesting as a solitary kidney, a staghorn calculus $4.5 \mathrm{~cm} \times 2.4 \mathrm{~cm}$ in size, and deformity of the pelvis. There were 2 points supporting the choice of PCNL. First, PCNL would be a preferable choice to manage the staghorn calculus based on the relevant guidelines. Second, the deformity and distortion of the urinary tract secondary to the pelvic deformity substantially increased the difficulty of performing RIRS, with conventional ureteroscopy made challenging by the need to pass through the deformed urinary tract. On the other hand, 3 points supported the choice of RIRS. First, its high safety level was particularly beneficial to preventing procedure-related kidney injury in the solitary kidney. Second, the multisession RIRS has a comparable stone-free rate to that of PCNL. Third, the application of flexible ureteroscopy could substantially mitigate the difficulty of navigating the urinary tract. The patient also expressed a clear preference for undergoing RIRS to minimize the risk of surgery-related renal damage. After thorough discussion, it was decided to proceed with multiple sessions of RIRS to minimize procedure-related renal damage. Flexible ureteroscopy was conducted to diminish the difficulty of passing through the deformed urinary tract. Three sessions of RIRS, together with holmium laser lithotripsy, were performed, and the staghorn calculus was completely removed in the solitary kidney, and a stonefree rate of $100 \%$ was achieved. The postoperative lab tests in each session, including those of creatinine and blood urea, were normal, indicating that RIRS may entail negligible renal collecting system injury even in cases with urinary tract deformity. Our experienced and skillful urologist was able to navigate the flexible ureteroscope through the deformed urinary tract without causing any bleeding. The serial perioperative CT and KUB revealed that the effect of RIRS in removing the renal stones was evident in each session, with removal being complete after the third session. In the present case, the patient presented with complex clinical conditions, including a solitary kidney, a staghorn calculus, and a marked pelvic deformity, which made the choice between PCNL and RIRS difficult. The unique situation of a solitary kidney raised particular concern concerning the safety of the procedure. A total of 3 sessions of RIRS were delivered to guarantee the thorough removal of the large stone, while flexible ureteroscopy was used to improve the likelihood of navigating through the deformed urinary tract. This case suggests that RIRS can be conducted effectively and safely to manage large kidney stones within a complex urinary system. In this rare case, a multisession RIRS with the use of a flexible ureteroscope successfully eliminated a staghorn calculus in a patient with a solitary kidney and deformed urinary tract, and maintained normal postoperative renal function. As the presence of a solitary kidney emphasized the importance of protecting the function of the only remaining kidney, RIRS was the preferable choice for 2 reasons. First, RIRS with multiple sessions was comparable to PCNL in terms of achieving an optimal stone-free rate for managing a staghorn calculus. Second, the successful navigation of the flexible ureteroscope through the deformed urinary tract made RIRS applicable in the presence of the urinary tract deformity. Consequently, our case indicates that, with technical advances, RIRS can be adopted in complex cases. However, the effectiveness and safety of RIRS still need to be explored in a large cohort.

However, the interpretations of this case study should be limited by some considerations. First, the case report consists of just 1 patient who was treated with RIRS for staghorn calculus in a solitary kidney. Therefore, our conclusions should be further validated in a prospective study with a large cohort. Second, some complications related to RIRS treatment for staghorn calculus in a solitary kidney, such as renal atrophy or renal impairment, may only be apparent in the long-term, Third, to achieve a 
high stone-clearance rate in our patient with staghorn calculus, more sessions of RIRS were needed as compared with PCNL, which resulted in a higher cost and longer procedure duration.

\section{Acknowledgments}

The authors would like to thank Dr. Lingyu Xu for her constructive advice regarding this article.

Funding: The study was supported by the Scientific Research and Cultivation Plan for High-Level Hospital Construction (No. hkuszh201902030).

\section{Footnote}

Reporting Checklist: The authors have completed the CARE reporting checklist. Available at https://dx.doi. org/10.21037/tau-21-192

Conflicts of Interest: All authors have completed the ICMJE uniform disclosure form (available at https://dx.doi. org/10.21037/tau-21-192). The authors have no conflicts of interest to declare.

Ethical Statement: The authors are accountable for all aspects of the work in ensuring that questions related to the accuracy or integrity of any part of the work are appropriately investigated and resolved. All procedures performed in studies involving human participants were in accordance with the ethical standards of the institutional and/or national research committee(s) and with the Helsinki Declaration (as revised in 2013). Written consent for all forms of personally identifiable data including biomedical, clinical, and biometric data was obtained from the patient.

Open Access Statement: This is an Open Access article distributed in accordance with the Creative Commons Attribution-NonCommercial-NoDerivs 4.0 International License (CC BY-NC-ND 4.0), which permits the noncommercial replication and distribution of the article with the strict proviso that no changes or edits are made and the original work is properly cited (including links to both the formal publication through the relevant DOI and the license). See: https://creativecommons.org/licenses/by-nc-nd/4.0/.

\section{References}

1. Türk C. Urolithiasis guidelines: retrospective view and perspectives. Urologe A 2016;55:1317-20.

2. Amaro CR, Goldberg J, Agostinho AD, et al. Metabolic investigation of patients with staghorn calculus: is it necessary? Int Braz J Urol 2009;35:658-61.

3. Preminger GM, Assimos DG, Lingeman JE, et al. Chapter 1: AUA guideline on management of staghorn calculi: diagnosis and treatment recommendations. J Urol 2005;173:1991-2000.

4. Türk C, Petř́k A, Sarica K, et al. EAU Guidelines on Interventional Treatment for Urolithiasis. Eur Urol 2016;69:475-82.

5. Bozkurt OF, Resorlu B, Yildiz Y, et al. Retrograde intrarenal surgery versus percutaneous nephrolithotomy in the management of lower-pole renal stones with a diameter of 15 to $20 \mathrm{~mm}$. J Endourol 2011;25:1131-5.

6. Scotland KB, Rudnick B, Healy KA, et al. Retrograde Ureteroscopic Management of Large Renal Calculi: A Single Institutional Experience and Concise Literature Review. J Endourol 2018;32:603-7.

7. Cohen J, Cohen S, Grasso M. Ureteropyeloscopic treatment of large, complex intrarenal and proximal ureteral calculi. BJU Int 2013;111:E127-31.

8. Hyams ES, Munver R, Bird VG, et al. Flexible ureterorenoscopy and holmium laser lithotripsy for the management of renal stone burdens that measure 2 to $3 \mathrm{~cm}$ : a multi-institutional experience. J Endourol 2010;24:1583-8.

9. Gadzhiev N, Malkhasyan V, Akopyan G, et al. Percutaneous nephrolithotomy for staghorn calculi: Troubleshooting and managing complications. Asian J Urol 2020;7:139-48.

10. Soylemez H, Penbegül N, Utangac MM, et al. Laparoscopy assisted percutaneous stone surgery can be performed in multiple ways for pelvic ectopic kidneys. Urolithiasis 2016;44:345-52.

11. Diri A, Diri B. Management of staghorn renal stones. Ren Fail 2018;40:357-62.

12. Shi X, Peng Y, Li L, et al. Renal function changes after percutaneous nephrolithotomy in patients with renal calculi with a solitary kidney compared to bilateral kidneys. BJU Int 2018;122:633-8.

13. Kuroda S, Fujikawa A, Tabei T, et al. Retrograde intrarenal surgery for urinary stone disease in patients with solitary kidney: A retrospective analysis of the efficacy and safety. Int J Urol 2016;23:69-73.

14. Bres-Niewada E. Solitary kidney - a clinical challenge for endourologist. Cent European J Urol 2016;69:96-7.

15. Bryniarski P, Paradysz A, Zyczkowski M, et al. A 
randomized controlled study to analyze the safety and efficacy of percutaneous nephrolithotripsy and retrograde intrarenal surgery in the management of renal stones more than $2 \mathrm{~cm}$ in diameter. J Endourol 2012;26:52-7.

16. Mousavi-Bahar SH, Mehrabi S, Moslemi MK.

Percutaneous nephrolithotomy complications in 671 consecutive patients: a single-center experience. Urol J 2011;8:271-6.

17. Pietropaolo A, Reeves T, Aboumarzouk O, et al. Endourologic Management (PCNL, URS, SWL) of Stones in Solitary Kidney: A Systematic Review from European Association of Urologists Young Academic Urologists and Uro-Technology Groups. J Endourol 2020;34:7-17.

18. Segura JW, Patterson DE, LeRoy AJ, et al. Percutaneous

Cite this article as: $\mathrm{Xu} \mathrm{Y,} \mathrm{Lu} \mathrm{Z,} \mathrm{Lan} \mathrm{Y}, \mathrm{Wu} \mathrm{D}$, Xiong L. Retrograde intrarenal surgery for a staghorn renal calculus in a patient with solitary kidney and urinary tract deformity: a case report. Transl Androl Urol 2021;10(8):3532-3539. doi: 10.21037/ tau-21-192 removal of kidney stones: review of 1,000 cases. J Urol 1985;134:1077-81.

19. Mariani AJ. Combined electrohydraulic and holmium: YAG laser ureteroscopic nephrolithotripsy of large (greater than $4 \mathrm{~cm}$ ) renal calculi. J Urol 2007;177:168-73.

20. Riley JM, Stearman L, Troxel S. Retrograde ureteroscopy for renal stones larger than $2.5 \mathrm{~cm}$. J Endourol 2009;23:1395-8.

21. Breda A, Ogunyemi O, Leppert JT, et al. Flexible ureteroscopy and laser lithotripsy for multiple unilateral intrarenal stones. Eur Urol 2009;55:1190-6.

22. Zeng G, Zhao Z, Wu W, et al. Combination of debulking single-tract percutaneous nephrolithotomy followed by retrograde intrarenal surgery for staghorn stones in solitary kidneys. Scand J Urol 2014;48:295-300. 